\title{
TRATAMENTO CONSERVADOR DAS ESTENOSES BENIGNAS DO ESÔFAGO ATRAVÉS DE DILATAÇOES. ANÁLISE DE 500 CASOS.
}

\author{
*N.A. Andreollo, L.R. Lopes, R. Inogutm, N.A. Brandalise, L.S. Leonardi \\ Trabalho realizado no Centro de Diagnóstico de Doenças Digestivas - GASTRO CENTRO \\ e Departamento de Cirurgia - Faculdade de Ciências Médicas da Universidade \\ Estadual de Campinas - UNICAMP - Campinas - SP
}

RESUMO - As estenoses benignas do esôfago (EBE) são complicações muito freqüentes, resultado de várias etiologias, a saber: refluxo gastro-esofágico, ingestão de agentes corrosivos, póscirurgias do esôfago, pós-radioterapia no tórax, pós-escleroterapia endoscópica de varizes do esôfago, ingestão de medicamentos, uso prolongado de cateter nasogástrico, compressão extrínseca e membranas esofágicas congênitas. As dilatações esofágicas são recomendadas no tratamento dessa complicação, empregando dilatadores de vários tipos e diâmetros, facilitando ao doente a ingestão alimentar.

OBJETTVos. Avaliação dos resultados e vantagens do tratamento conservador das EBE através de dilatações esofágicas realizadas ambulatorialmente com auxílio da endoscopia digestiva flexível.

Métodos. No período de $198 \mathrm{I}$ a 1999 foram tratados, conservadoramente e seguidos no Gastrocentro - UNICAMP, 500 doentes com EBE, através de um Programa de Dilatações Esofágicas instituído para cada caso. A maioria era do sexo masculino $(59,2 \%)$ e a faixa etária mais acometida encontra-se entre 31 anos e 60 anos, compreendendo $52,8 \%$ dos pacientes em estudo.

As estenoses mais prevalentes foram as estenoses pépticas $(30,4 \%)$, cáusticas $(23,6 \%)$, de anastomoses $(23,2 \%)$, por megaesôfago $(8,0 \%)$ e por uso prolongado de cateter nasogástrico (6,4\%), perfazendo um total de $91,6 \%$ das EBE.
Quanto ao número de procedimentos, $94,2 \%$ dos casos foram submetidos a no máximo 25 dilatações do esôfago. Em 95,6\% deles foram utilizados dilatadores com diâmetro entre $10,5 \mathrm{~mm}$ e $16,0 \mathrm{~mm}$. A duração do tratamento foi até 24 meses em 76,2\% dos casos. Perfurações esofágicas ocorreram em seis doentes (I,2\%), sem mortalidade.

Resultados. Foram considerados bons em $76,2 \%$, regulares em $18,2 \%$ e maus em $5,6 \%$ dos doentes. 0 sucesso do tratamento variou conforme a etiologia da estenose, ocorrendo bons resultados em $81,0 \%$ das estenoses pépticas, em $66,1 \%$ das estenoses cáusticas e em $82,7 \%$ das estenoses de anastomoses. A falha do tratamento conservador ocorreu em $9,3 \%$ das estenoses cáusticas, $4,3 \%$ das estenoses de anastomose e 3,9\% das estenoses pépticas. A estenose cáustica propiciou a falha maior da terapêutica conservadora em relação aos demais.

CONCLUSÄO. 0 tratamento conservador, através de dilatações orientadas por fio-guia (Savary-Gilliard e Eder Puestow), é a primeira escolha nas EBE; é eficaz a longo prazo, com índice mínimo de complicações e o tratamento cirúrgico é apenas indicado se não for possível realizar as dilatações.

UnITERMOS: Estenoses benignas do esôfago. Dilatadores de esôfago. Dilatações. Esôfago.

\section{INTRODUÇÃO}

A estenose benigna do esôfago (EBE) resulta de uma lesão intensa da mucosa do órgão com conseqüente espessamento de suas camadas mucosa, submucosa e mus-

*Correspondência:

Nelson Adami Andreollo

Rua Francisco Humberto Zuppi, 1.234 - Cidade

Universitária - Campinas - SP - Cep: 13083-350

Tel.: (19) 3289-2296-E-mail: nandreollo@ mailbr.com.br cular, evoluindo até para fibrose. Não é uma doença primária, mas uma complicação secundária à ação de um fator exógeno ou de uma doença já existente!

O paciente com EBE apresenta uma tríade de sintomas: disfagia progressiva, iniciando-se com alimentos sólidos e progredindo até líquidos, regurgitação e azia².

A formação das EBE pode ser desencadeada por vários fatores, sendo que os principais são: refluxo gastro-esofágico
(RGE), ingestão de agentes corrosivos (ácidos ou básicos), anastomoses esofágicas, tais como esofagogastroplastia, esofagocolopastia e esofagojejunostomia, uso prolongado de cateter nasogástrico, que pode estar associado ao refluxo gastro-esofágico, pós-escleroterapia endoscópica de varizes do esôfago, pós-esofagomiotomia para o tratamento do megaesôfago ou da acalasia idiopática, membranas esofágicas congênitas, pós-radioterapia no tórax, medica- 
mentosa por tetraclina, doxiciclina, quinidina, antiinflamatórios não-esteroidais (Al$\mathrm{NE}$ ) e cloridrato de potássio e compressões extrínsecas ${ }^{3-11 .}$

Os objetivos da terapia no tratamento das estenoses esofágicas benignas são o alívio da disfagia e a prevenção da recorrência das estenoses.

Os métodos atuais de tratamento consistem na intervenção cirúrgica, dilatações esofágicas e o uso de próteses metálicas (stents), sendo que esses dois últimos constituem o tratamento conservador das EBE, os quais, sem dúvida, têm diminuído as indicações para o tratamento cirúrgico 6,12,13.

A cirurgia é reservada para os casos de EBE refratárias ao tratamento com dilatações. É de baixa morbimortalidade se o paciente encontra-se em boas condições de saúde, porém em se tratando de idosos, cardiopatas, pneumopatas ou desnutridos, o risco de complicações é elevado ${ }^{2,14}$.

As dilatações esofágicas têm a finalidade de expandir a estenose e aumentar o seu diâmetro interno, sendo uma técnica utilizada desde o século 16. No início do século 20 , as estenoses esofágicas já podiam ser detectadas através do exame radiológico, graças a descoberta de Röentgen. Neste século, foram desenvolvidos os dilatadores, instrumentos de biopsias e endoscópios flexíveis adequados para a avaliação do tubo gastrointestinal ${ }^{\prime, 14}$.

Os dilatadores mais freqüentemente utilizados atualmente são: dilatadores de borracha contendo mercúrio no interior (Malloney e Hurst), dilatadores guiados por fio-guia (Savary-Gilliard, Celestin, Eder-Puestow e Plummer-Jackson) e balões hidrostáticos ou pneumáticos de poliure$\operatorname{tano} 0^{14,15}$.

As dilatações esofágicas são os primeiros procedimentos indicados diante de portadores de estenoses esofágicas de quaisquer etiologias. $\mathrm{O}$ emprego rotineiro de equipamentos de endoscopia digestiva fle- xíveis, associados aos fios-guia de aço, passados através do canal de biopsia do apareIho, transpondo a estenose e atingindo a luz gástrica, propicia muita segurança ao método e trouxe grandes benefícios aos doentes, dispensando o uso, antigamente obrigatório, de gastrostomias para a realização das dilatações. O fio de aço assim introduzido servirá como guia seguro para orientar a passagem de dilatadores denominados rígi$\operatorname{dos}^{2,14,16}$.

O objetivo do presente trabalho é avaliar os resultados e vantagens do tratamento conservador de estenoses benignas do esôfago de várias etiologias, através de dilatações esofágicas ambulatoriais, com auxílio da endoscopia digestiva flexível.

\section{Métodos}

No Serviço de Endoscopia Digestiva do Gastrocentro-UNICAMP foram tratados e seguidos, nos últimos dezoito anos ( 198 I a 1999), 500 doentes portadores EBE, através de dilatações esofágicas. As informações foram obtidas dos prontuários dos doentes, sendo incluídos somente os doentes que completaram o tratamento e tiveram seguimento ambulatorial.

A faixa etária desta casuística variou de 13 anos até 90 anos (idade referente ao início do tratamento), sendo mais freqüente entre 3 I e 60 anos (52,8\%). A sua distribuição quanto ao sexo é mostrada na Tabela 1.

O tratamento e seguimento dos doentes foi a longo prazo na forma de um Programa de Dilatações Esofágicas, estabelecido individualmente para cada caso.

As etiologias das estenoses são mostrados na Tabela 2.

As estenoses pépticas mais freqüentes foram no terço distal do esôfago, somando I 48 casos (29,6\%), entretanto, em quatro casos (0,8\%) foram no terço proximal.

Nos portadores de megaesôfago, 16 doentes (40\%) apresentavam estenoses pós-cardioplastias e os demais 24 casos (60\%) não foram operados.

As estenoses de anastomoses mais freqüentes foram esôfago-gástrica cervical, totalizando 100 casos $(86,5 \%)$, seguidas pela esôfago-jejunal - cinco casos (4,4\%), esôfago-cólica cervical - quatro casos (3,5\%), esôfago-gástrica intratorácica - três casos (2,6\%), faringo-gástrica cervical dois casos (1,7\%), esôfago-gástrica abdominal com grampeador - dois casos $(1,7 \%)$, esôfago-gástrica abdominal (manual) - um caso $(0,8 \%)$ e faringo-cólica cervical - um caso $(0,8 \%)$.

Dentre o total de 189 doentes com estenoses esofágicas secundárias a algum tipo de procedimento cirúrgico, em 96 casos (50,8\%) foram por doenças benignas e nos demais 93 casos $(49,2 \%)$ por doenças malignas.

As estenoses secundárias ao uso prolongado de cateter nasogástrico foram todas no terço distal do esôfago, com tempo variável de três a 32 dias de manutenção do cateter. Em $27 \operatorname{casos}(84,4 \%)$ a sua permanência foi devido a cirurgias abdominais eletivas ou de urgência e em cinco casos (I5,6\%) foram por doenças neurológicas.

As estenoses pós-radioterapia no tórax foram indicadas devido: mama - cinco casos (45,4\%), neoplasia de traquéia e brônquios - quatro casos (36,4\%), esôfago - um caso $(9,1 \%)$ e mediastino - um caso $(9,1 \%)$, com doses variando de 500 a $700 \mathrm{Cy}$.

As estenoses pós-escleroterapia de varizes foram secundárias ao emprego de oleato de etanolamina em quatro casos $(66,7 \%)$ e álcool absoluto em dois casos (33,3\%), com número de aplicações variando de 6 a 14 vezes.

As membranas esofágicas diagnosticadas foram todas no terço proximal do esôfago. E as compressões extrínsecas foram secundárias a doenças mediastinais.

As dilatações esofágicas foram realizadas ambulatorialmente. O preparo foi se- 
melhante ao utilizado para endoscopia digestiva alta, com anestesia local na orofaringe empregando lidocaína aspersão a 10\% e sedação com diazepam 10 mg IV, eventualmente sendo utilizado meperidina. $O$ procedimento iniciava-se com endoscopia digestiva alta utilizando-se fibroendoscópios flexíveis, o local da estenose localizado e o seu diâmetro interno avaliado. Em seguida, passado o fioguia de aço pelo canal de biopsia do fibroscópio, introduzido através da estenose, preferencialmente o mais distal possível no estômago. $O$ aparelho era retirado e mantido o fio-guia no local, o qual orientava para a introdução e passagem dos dilatadores. Os dilatadores utilizados foram o de Eder-Puestow e o de Savary-Gilliard, sendo os diâmetros de ambos idênticos.

O dilatador de Eder-Puestow foi preferencialmente empregado nas estenoses com muita fibrose, tais como as de cáusticos e de anastomoses. A opção pelo dilatador de Savary-Gilliard foi quando era necessário realizar dilatações mais suaves, por exemplo, nas estenoses pépticas.

O intervalo de tempo entre a sessões era determinado, considerandose a etiologia da estenose e a facilidade de ingestão alimentar. Nas estenoses graves com disfagia intensa, o doente realizava as dilatações a cada sete dias, passando depois a 14, 21 ou 28 dias, conforme melhorava a ingestão alimentar. $O$ doentes com diâmetro interno do esôfago próximo do normal, retornavam a cada seis meses ou eventualmente uma vez ao ano para calibração da estenose.

Perfurações esofágicas ocorreram em seis casos $(1,2 \%)$ de estenoses cáusticas, sendo quatro casos no esôfago torácico e dois casos no abdominal, porém todos foram prontamente tratados.

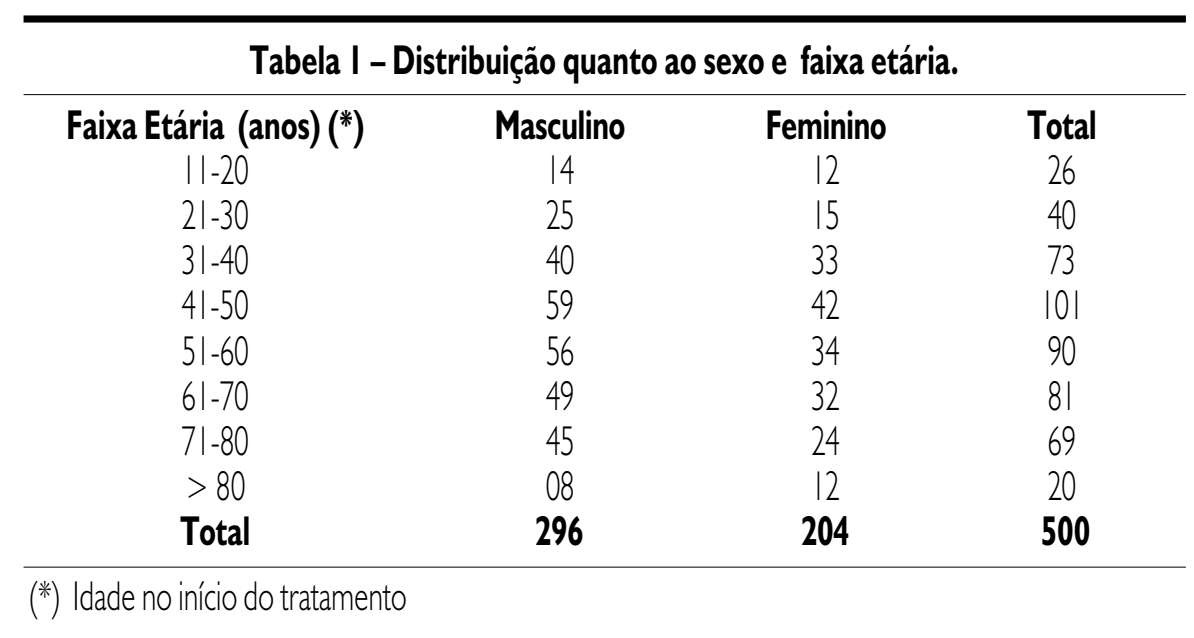

\section{Tabela 2 - Etiologias das estenoses benignas do esôfago.}

\begin{tabular}{|c|c|c|}
\hline Etiologias & n. ${ }^{\circ}$ de casos & $\%$ \\
\hline EstenosePéptica & 152 & 30,4 \\
\hline Estenose Cáustica & 118 & 23,6 \\
\hline Estenose deAnastomoses & 116 & 23,2 \\
\hline Megaesôfago & 40 & 8,0 \\
\hline Estenose por uso prolongado de cateter nasogástrico & 32 & 6,4 \\
\hline Estenose pós-radioterapia no tórax & 07 & 1,4 \\
\hline Esclerose pós-laringectomia & |l & 2,2 \\
\hline Estenose pós-escleroterapia de varizes & 06 & 1,2 \\
\hline Membranas esofágicas & 06 & 1,2 \\
\hline Compressões extrínsecas & 04 & 0,8 \\
\hline Estenose pós-vagotomia supra-seletiva & 02 & 0,4 \\
\hline Estenose pós-infecção HPV & 01 & 0,2 \\
\hline Outros $(*)$ & 05 & 1,0 \\
\hline Total & 500 & 100,0 \\
\hline
\end{tabular}

\section{Resultados}

Os resultados e benefícios obtidos com as dilatações foram avaliados considerando-se: a etiologia da estenose, as cirurgias anteriores, o número de dilatações, o maior diâmetro do dilatador utilizado, o tempo de tratamento e a melhora da disfagia.

A Tabela 3 mostra o tempo de dura- ção do tratamento. Notar que 50,6\% dos casos necessitaram de aproximadamente seis meses de tratamento, em 25,6\% deles o tempo de tratamento atingiu 24 meses e 1।,6\% tiveram mais de 60 meses de seguimento.

A Tabela 4 mostra o número de dilatações realizadas. Em $60 \%$ dos casos foram necessárias até cinco sessões de dilatações. 
Em 26,6\% dos casos o número de sessões variou de 6 a 15 vezes, sendo que apenas aproximadamente $1 \%$ dos casos necessitou de mais de 50 sessões.

Quanto ao maior diâmetro das ogivas ou dilatadores utilizados, foram obtidos: 6,0 a 8,0 em 5 casos (1\%); 8,5 a 10,0 em 17 casos (3,4\%); 10,5 a 12,0 em 52 casos $(10,4 \%) ; 12,5$ a 14,0 em 168 casos (33,6\%); 14,4 a 16,0 em 258 casos (51,6\%). Portanto, em 85,2\% dos doentes foi possível chegar ao dilatador com diâmetro aproximado de 14 mm ou maior, o qual possibilita a ingestão da maioria dos alimentos sólidos bem mastigados.

A maioria dos doentes dessa casuística referiu melhora na ingestão alimentar com as dilatações, sendo o resultado considerado bom em 38I casos $(76,2 \%)$ e regular em 9 I (I 8,2\%). Em 28 doentes (5,6\%) não foi possível realizar as dilatações e ocorreram perfurações ou mantiveram dificuldade de ingestão até mesmo de alimentação líquida, sendo encaminhados para tratamento cirúrgico, desde que reunissem condições clínicas satisfatórias para tal.

Os dois doentes que apresentaram perfuração de esôfago abdominal foram imediatamente operados, tendo sido realizado sutura da lesão e proteção com o fundo gástrico. Dentre os quatro outros com perfuração de esôfago torácico, em dois foi possível o tratamento clínico, incluindo jejum via oral, alimentação através de cateter nasoentérico e antibioticoterapia. Nos demais foi necessário optar por esofagostomia cervical terminal seguido de drenagem torácica fechada e gastrostomia, além de antibioticoterapia de amplo espectro. Não ocorreram óbitos.

A Tabela 5 relaciona os resultados das dilatações conforme as principais etiologias de EBE, bem como o número de sessões de dilatações. $O$ tratamento foi considerado com sucesso quando o doente referiu resultado bom ou regular. A falha do trata-

Tabela 3 - Duração do tratamento dos pacientes portadores de EBE.

\begin{tabular}{ccc}
\hline Duração do tratamento (meses) & $\mathbf{n}^{\circ}{ }^{\circ}$ de casos & $\%$ \\
$<6$ & 253 & 50,6 \\
$06-12$ & 65 & 13,0 \\
$12-18$ & 42 & 8,4 \\
$18-24$ & 21 & 4,2 \\
$24-30$ & 12 & 2,4 \\
$30-36$ & 13 & 2,6 \\
$36-42$ & 13 & 2,6 \\
$42-48$ & 06 & 1,2 \\
$48-54$ & 11 & 2,2 \\
$54-60$ & 06 & 1,2 \\
$>60$ & 58 & 11,6 \\
\hline Total & & 100,0 \\
\hline
\end{tabular}

mento ocorreu quando o doente continuou ingerindo apenas alimentação líquida, ocorreu perfuração ou foi recomendado para tratamento cirúrgico.

Observar na Tabela 5 que o sucesso no tratamento das estenoses pépticas e de anastomoses foi muito semelhante. Nas estenoses cáusticas a porcentagem de sucesso foi inferior, bem como a média de dilatações realizadas foi significativamente superior.

\section{Discussão}

O refluxo gastro-esofágico prolongado é a causa mais comum da EBE, que se localiza na maioria das vezes na junção esôfago-gástrica ${ }^{4}$.

A maioria das casuísticas relatam que as estenoses pépticas estão presentes em freqüências variáveis de $8 \%$ a 20\% dos portadores de esofagites. Embora seja mais racional pensar que os doentes com estenoses pépticas apresentariam refluxo mais grave e exposição mais prolongada ao conteúdo cloridro-péptico, não existem informações concretas sobre o tempo necessário para ocorrer a estenose $e^{4,16}$.
Tabela 4 - Número de dilatações realizadas.

\begin{tabular}{ccc}
$\mathbf{N} .{ }^{\circ}$ de Dilatações & $\mathbf{n .}^{\circ}$ de casos & $\%$ \\
$0 \mid-05$ & $30 \mid$ & 60,2 \\
$06-10$ & $9 \mid$ & $\mid 8,2$ \\
||$-\mid 5$ & 42 & 8,4 \\
$\mid 6-20$ & 20 & 4,0 \\
$2 \mid-25$ & 17 & 3,4 \\
$26-30$ & 06 & 1,20 \\
$3 \mid-35$ & || & 2,40 \\
$36-40$ & 04 & 0,80 \\
$4 \mid-45$ & $\mid$ & 0,2 \\
$46-50$ & 03 & 0,60 \\
$>50$ & 04 & 0,80 \\
\hline Total & $\mathbf{5 0 0}$ & $\mathbf{1 0 0 , 0}$ \\
\hline
\end{tabular}

Esta complicação ocorre em ambos os sexos indistintamente, desde o jovem até o muito idoso, porém afaixa etária predominante é acima dos 60 anos. A literatura tem registrado elevação da ocorrência do refluxo gastro-esofágico nos idosos, mesmo assintomáticos, explicado pela diminuição da velocidade e da amplitude das ondas de contrações esofágicas primárias nesta faixa etária 4 . 
A associação de estenoses pépticas com adenocarcinoma é um assunto ainda controverso. É importante que, diante de portadores de estenoses orgânicas de esôfago, a presença de um carcinoma precisa ser afastada ou confirmada imediatamente, através de biopsias endoscópicas, repetidastantasvezes quantas forem necessárias. A associação entre epitélio de Barretteadenocarcinoma pareceser clara e conhecida e o elemento comum é o refluxo gastro-esofágico. Nessa associação tem sido registrado que mais de $80 \%$ dos doentes têm estenoses pépticas do esôfago. Faltam ainda informações para correlacionar a duração dos sintomas de refluxo, a estenose péptica e a transformação maligna da mucosa ${ }^{4,17,18}$.

No tratamento conservador das estenoses pépticas são mais freqüentemente utilizados dois tipos de dilatadores: o de SavaryGilliard'19 ou os balões hidrostáticos ou pneumáticos ${ }^{20}$. Estudo realizado por Saeed et $\mathrm{al}^{5}$, para comparar a eficácia desses dois dilatadores, concluiu que ambos são eficazes em promover oalívio da disfagia. O balão apresenta uma vantagem a longo prazo queéa menor taxa de recidiva, além da necessidade de menor número de procedimentos para atingir um diâmetro adequado da luz esofágica e melhor tolerância do paciente ao balão.

As estenoses de origem cáustica são freqüentes em todo território nacional, sendo que a ingestão desse agente leva a estenose de esôfago de 10\% a 33\% dos casos registrados ${ }^{4}$. O mais comum é a própria soda cáustica para uso doméstico, porém, outros produtos tais como amoníaco, ácido nítrico, ácido muriático, ácido clorídrico, soluções para baterias e outros produtos de limpeza contendo substâncias cáusticas também são ingeridos. A evolução para estenose geralmente ocorre nas primeiras 2-8 semanas, freqüentemente é de grande extensão e persiste por longo tempo ${ }^{4,6}$.

O tratamento inicial é a tentativa de dilatações e caso não ocorra melhora da

Tabela 5 - Resultados das dilatações conforme as principais etiologias de estenose $\mathrm{e}$ número de sessões de dilatações.

\begin{tabular}{lcccccc}
\hline Estenose & Bom & $\begin{array}{c}\text { Resultados } \\
\text { Regular }\end{array}$ & Mau & Média de sessões & $\begin{array}{c}\text { Sucesso } \\
\text { Tratamento(\%) }\end{array}$ & $\begin{array}{c}\text { Falha } \\
\text { Péptica }\end{array}$ \\
\hline & 124 & 22 & 06 & 06 & 81,0 & 3,9 \\
Cáustica & 78 & 29 & 11 & 15 & 66,1 & 9,3 \\
Anastomose & 96 & 15 & 05 & 06 & 82,7 & 4,3 \\
\hline
\end{tabular}

disfagia, com queda do estado geral e emagrecimento, o tratamento cirúrgico deverá ser logo indicado. Com o progresso e avanços trazidos pelos novos dilatadores e métodos de dilatações associados à endoscopia flexível, apenas um dentre aproximadamente 20 doentes que ingerem cáusticos têm necessidade de tratamento cirúrgico para reconstrução do trânsito digestivo e retorno à alimentação por via oral ${ }^{17,21}$.

É importante lembrar que o doente portadordeestenose cáustica deveráter acompanhamento médico e endoscópico por longos anos, ou durante praticamente toda a sua vida, uma vezque com o passar dotempoa disfagia poderá retornar, secundária a novo estreitamento e reestenose, secundária a RGE, o qual se torna mais freqüente neste grupo. Além disso, a literatura descreve a ocorrência mais freqüente de carcinoma epidermóide nos doentes que ingeriram produtos cáusticos, principalmente após 30 a 40 anos do incidente. Esta associação, segundo algumas publicações, não ultrapassa $3 \%$ a $5 \% 17$.

As estenoses de anastomoses esofágicas na maioria das vezes são secundárias a fístulas ou à técnica cirúrgica empregada. Geralmente são relativamente fáceis de dilatar, com risco pequeno de complicações quando estiverem localizadas na região cervical, embora seja necessário número não desprezível de sessões de dilatações para se atingir um diâmetro intraluminar razoável. É mais freqüente nas esofagogastroplastias que nas esofagocolo- plastias $5,8,22,23,24$. Heitmiller et al. ${ }^{24}$ relatam a necessidade de dilações em $26 \%$ nos primeiros 6 meses de pós-operatório das esofagogastroplastias cervicais.

A estenose esofágica do megaesôfago ou da acalasia e a recidiva da disfagia pósesofagomiotomia está principalmente relacionada a RGE, porém pode ter outras etiologias, tais como: recidiva da acalasia secundária a progressão da doença, a fibrose pós-cirurgia e raramente carcinoma do esôfago. O tratamento baseia-se no uso de balão ou dilatador de Savary-Gilliard, obtendo-se bons resultados com ambos os procedimentos ${ }^{9}$. A reoperação é difícil devido a presença de tecido cicatricial e aderências da primeira cirurgia, sendo indicada quando as dilatações não tiverem sucesso. Resumindo, em pacientes de alto risco (idosos e indivíduos com doenças secundárias graves ), a escolha pelo tratamento conservador é mais racional, enquanto em pacientes de baixo risco e jovens, o tratamento cirúrgico é o mais indicado 9,14,25.

As estenoses do esôfago pós-radioterapia para o tratamento de câncer da cavidade torácica e de cabeça e pescoço (laringe, faringe e língua), associado ou não a intervenção cirúrgica surgem como resultado da fibrose causada pelo processo inflamatório local. Podem ser dilatadas com o dilatador Savary-Gilliard, obtendo-se alívio da disfagia em mais de 66\% dos $\operatorname{casos}^{10,22}$.

Uma das complicações da escleroterapia endoscópica de varizes éa estenose esofágica ${ }^{26}$. 
Nessa casuística compreendeu I,2\% das EBE, sendo frequentemente localizada, e motivo de sintomas disfágicos significativos.

Várias publicações relatam risco elevado, variando entre $14 \%$ a $78 \%$, de desenvolver EBE em indivíduos que consomem drogas antiinflamatórias não-esteroidais (AINE). Nesse grupo de risco estão incluídos as doenças tratados com AINE como as doenças músculo-esqueléticas".

A estenose mais comum nessa casuística foi a estenose péptica (30,4\%), seguida da estenose cáustica (23,6\%), estenose de anastomose $(23,2 \%)$, megaesôfago (8,0\%), estenose por uso prolongado de cateter nasogástrico (6,4\%) e outras causas (8,4\%).

Analisando-se as etiologias das estenoses nas casuísticas de outros autores ${ }^{18,21,27}$, observase que a estenose péptica e de anastomose são as principais causas de EBE, enquanto a estenose cáustica parece não ser tão prevalente, provavelmente porque acomete extensamente 0 esôfago, diminuindo seu calibre, dificultando a sua dilataçãa, acarretando elevada taxa de falha terapêutica em relação as outras etiologias, e assim os doentes são freqüentemente tratados cirurgicamente. As opções mais empregadas sãoa esofagogastroplastia e a esofagocoloplastia. Otratamento cirúrgico traz risco de mortalidade entre 5,7\% a 20\% e morbidez não desprezível devido complicações pós-operatórias ${ }^{8,24}$. A primeira escolha para o tratamento da estenose cáustica continua sendo a dilataçãa do esôfago e com resultados satisfatórios conforme foi também observado nesta casuística.

Nesta pesquisa a estenose de anastomose esôfago-gástrica cervical (86,5\%) foi a mais freqüente em comparação às estenoses de anastomose esôfago-cólica (3,5\%). A substituição do esôfago pelo estômago tem algumas vantagens: é cirurgia realizada em uma etapa, requer apenas uma anastomose cervical, apresenta boa elasticidade, adequado suprimento sangüíneo, alto potencial de cicatrização, boa resistência a pressão intratorácica negativa e poucas complicações como úlcera péptica. A longo prazo podem surgir alguns problemas como a regurgitação, esofagite grave e a síndrome de "dumping". Por outro lado, Yararbai et al. ${ }^{8}$ relatam que, considerando-se os resultados a longo prazo, a substituição pelo cólon apresenta meIhores resultados se sua irrigação for adequada, pois promove um peristaltismo uniforme por todo segmento da alça.

Dentre os mais empregados, os dilatadores Eder-Puestow, com as suas ogivas metálicas de calibres progressivos e haste flexível, propiciam um benefício indiscutível no alívio da disfagia, na grande maioria dos doentes, já nas primeiras sessões de dilatações. São dilatadores de baixo custo, elevada durabilidade e indicados nas estenoses graves com fibrose local, por exemplo nas estenoses secundárias a ingestão de produtos cáusticos e de anastomoses. Na prática, podem ser empregados com quaisquer tipos de estenoses, dispensando a presença da radioscopia para mostrar a localização do fio-guia. O procedimento todo pode se realizado nos adultos e jovens mediante uma sedação endovenosa combenzodiazepínicos e em regime ambulatorial, dispensando a internação hospitalar. Em crianças, entretanto, na maioria das vezes, seránecessário realizar o procedimento sob anestesia geral. Apesar do Eder-Puestow ser constituído de metal e ser necessário aplicar uma força maior para permitir a passagem das ogivas através das estenoses, a incidência de perfurações é baixa e sua eficácia é elevada 15,20,28,29.

Outro tipo de dilatador considerado rígido é o dilatador de Celestin, um longo tubo de polivinil com a extremidade afilada e canal central, passado também com o auxílio de fio guia de aço. Embora não seja muito utilizado, porque está disponível no mercado somente com diâmetros entre 12 mme $18 \mathrm{~mm}$, étambém útil em calibrações esofágicas e estenose não graves ${ }^{28}$.

Em anos recentes, foram introduzidos os dilatadores de Savary-Gilliard, com o mesmo aspecto que os de Celestin, porém muito mais flexíveis por serem à base de silicone e com opções de diversos calibres progressivos, desde $5 \mathrm{~mm}$ até $20 \mathrm{~mm}$. Seu emprego também é bem amplo, semelhante ao dos dilatadores de Eder-Puestow, com o inconveniente de serem de custo bem mais elevado e menor durabilidade que as ogivas metálicas 5, 14,18,27.

Dentre os dilatadores considerados não rígidos, devem ser citados os dilatadores de Malloneye Hurst, bem maleáveis ede borracha, contendo mercúrio em seu interior. Dispensam tanto o uso de fios guia de aço como a sedação e a endoscopia digestiva e têm elevada segurança. Não estão indicados em estenoses graves e são de durabilidade limitadal, 14 .

Os balões pneumáticos são outra opção quando ocorre apenas edema e subestenoses, portanto são indicados em estenoses não graves. São de custo elevado, baixa durabilidade e não têm ampla aplicabilidade como os dilatadores anteriormente citados. Nos doentes apresentando estenoses nãofibróticas são recomendados tanto quanto os dilatadores Malloney e Hurst, mas menos úteis que os dilatadores guiados por fio de aço ${ }^{5,20,25}$. Ikeya et al. ${ }^{25}$ enfatizam que o uso de balões nas estenoses benignas é efetivo quando a extensão é menor que $12 \mathrm{~mm}$.

O tratamento conservador das EBE através de dilatações contínuas e periódicas tem sido preconizado por vários autores, uma vez que é preferível o doente permanecer com o próprio órgão, a ter algum substituto que não the permita ter boa ingestão e digestão alimentar $2,3,6,15,17,21,27,30$.

O índice de perfurações esofágicas de I,2\% está em conformidade com a literatura, que registra freqüências de 0,3\% a 3,5\% na maioria das publicações ${ }^{14,17}$. Não ocorreu mortalidade porque o diagnóstico da perfuração foi feito precocemente, sendo o tratamento prontamente instituído.

Assim, Lanza et al. ${ }^{2}$ demonstram que 0 tratamento conservador das EBEcom auxilioda endoscopia é um método eficaz na maioria dos 
casos, permitindo avaliar o tipo de lesão presente (esofagite, úlcera, epitélio de Barrett ou câncer) ou se há associações com outras doenças como hérnia hiatal. O tratamento cirúrgico das EBE tem baixa mortalidade em doentes sem risco, porém, infelizmente, muitos são idosos e freqüentemente apresentam cardiopatia ou pneumopatia associadas. Além disso, deve-se considerar as complicações pós-operatórias, tais como a deiscência e fístula da anastomose, necrose de alça, mediastinite e mesmo estenose de anastomose tardia, a qual é possível de ocorrer. As dilatações podem ser realizadas sem mortalidade e com mínimo de morbidez a longo prazo ${ }^{14,29}$.

Na presente casuística, as dilatações foram realizadas com os dilatadores de Savary-Gilliard eEder-Puestow, com o auxílio da endoscopia, na forma de um Programa de Dilatações individual para cada caso e os resultados foram considerados muito satisfatórios.

Existem poucos estudos demonstrando quais os melhores tipos de dilatadores e suas respectivas eficácias sobre as diversas etiologias das EBE. Segundo revisão de Dumon et al ${ }^{19}$, feita em vários Serviços de Endoscopia, o Savary-Gilliard foi considerado o mais eficaz, seguro e de melhor manuseio que o Eder-Puestow. Cox et al. ${ }^{20} \mathrm{com}$ pararam a eficácia das ogivas e dos balões guiados endoscopicamente nas estenoses pépticas, mas com resultados controversos, e sugerem uma discreta eficácia das ogivas sobre os balóes. Por outro lado, Saeed et a $\left.\right|^{5,16}$ concluíram que o balão apresenta vantagens sobre as ogivas com menor taxa de recidiva, necessidade de menos procedimentos para atingir um diâmetro. Em vários serviços e instituições o uso do dilatador Eder-Puestow tem sido abandonado a favor do dilatador Savary-Gilliard, provavelmente por ser de mais fácil manipulação e melhor tolerabilidade 4, $15,21,28,31,32$. .

O Serviço de Endoscopia Digestiva do Gastrocentro não utiliza de rotina a radioscopia para as dilatações. Fleischer et al. ${ }^{20}$ e Kadakia et al. ${ }^{31}$ também já demonstraram que o emprego destes dilatadores associados ao fio-guia de aço é um método seguro, sem necessidade de monitorização através da radioscopia, desde que o endoscopista obedeça com rigor todas as normas estabelecidas para o procedimento.

O diâmetro das ogivas e velas utilizados nos pacientes desse estudo variaram entre 6,5 $\mathrm{mm}$ a $16 \mathrm{~mm}$. Patterson et al. ${ }^{18} \mathrm{e}$ Wo et al. ${ }^{4}$ acreditam que o uso de dilatadores com calibre superiora 45 French ( 15 mm) nãoéjustificável, pois não há a diminuição da taxa de recidiva e nem da necessidade de dilatações subsequentes. Broor et al. ${ }^{17}$ também recomendam as dilatações até o diâmetro aproximado de 15 mm para tratamento adequado.

A média de sessões de dilatações necessária para alcançar o alívio da disfagia foi de seis sessões para a estenose péptica (variando entre 0 l a 38 sessões), I 5 sessões para a estenose cáustica (variando entre 01 a 73 sessões) e seis sessões para a estenose de anastomose (variando entre 01 a 32 sessões).

O sucesso do tratamento conservador é avaliado através da melhora da disfagia relatada pelo doente. No GastrocentroUNICAMP a taxa de sucesso com bons resultados foi de $76,2 \%$, enquanto que nos trabalhos de Pereira-Lima et al. ${ }^{27}$, Wesdorp et al. ${ }^{21}$ e Patterson et al. ${ }^{18}$ foram de $66,5 \%$, $88,0 \%$ e $84,5 \%$, respectivamente. No primeiro trabalho os autores utilizaram os dilatadores Savary-Gilliard e Eder-Puestow, e no segundo e terceiro utilizaram os de Eder-Puestow e de mercúrio.

A etiologia da estenose também determina o sucesso do tratamento. Assim, este estudo demonstra bons resultados em $81,0 \%$ das estenoses pépticas, em 66,1\% das estenoses cáusticas e em 82,7\% das estenoses de anastomose (Tabela 5), resultados bem semelhantes aos referidos na literatura consultada.

A falha do tratamento conservador ocorreu em 9,3\% das estenoses cáusticas,
4,3\% das estenoses de anastomose e 3,9\% das estenoses pépticas. A estenose cáustica apresentou a falha maior da terapêutica conservadora em relação aos demais, provavelmente pelas razões anteriormente citadas.

Pode-se concluir que o tratamento conservador através de dilatações orientadas por fio-guia (Savary-Gilliard e Eder Puestow) é a primeira escolha nas EBE, é eficaz a longo prazo, com índice mínimo de complicações, e o tratamento cirúrgico é apenas indicado se não for possível realizar as dilatações.

\section{Agradecimentos}

À Fundação de Amparo à Pesquisa do Estado de São Paulo - FAPESP

\section{Conservative treatment of beNIGN ESOPHAGEAL STRICTURES BY DILATIONS. ANA- LYSIS OF $\mathbf{5 0 0}$ CASES.}

The benign esophageal stenoses (BES) are common complications owing to many etiologies: gastroesophageal reflux, ingestion of corrosive agents, esophageal surgery, radiotherapy, postendoscopic variceal sclerotherapy, drug ingestion, prolonged nasogastric intubation, extrinsic compression and esophageal webs.

Esophageal dilatations are worldwide recommended to treat this complication, employing dilators of many types and diameters and facilitating the food ingestion.

PURPOSE. Evaluation of the results and advantages ofthe conservative treatment ofthe BES using esophageal dilatations, in outpatient service of upper digestive endoscopy.

METHODS. During the period from 1981 to 1999,500 patients with BES were treated and followed up at the Gastrocenter UNICAMP, in an individually Program of Esophageal Dilatation for each case. The highest number of cases was under ages from 31 to 60 years old (52,8\%), and males $(59,2 \%)$. The most predominant etiologies were: peptic stenosis (30,4\%), caustic inges- 
tion (23,6\%), anastomosis (23,2\%), megaesophagus $(8,0 \%)$ and prolonged nasogastric ingestion entubation (6,4\%), totalizing 91,6\% of the BES. Most of patients (94,2\%) were submitted to the maximum of 25 dilations. Dilatorsfrom 10,5 to $16 \mathrm{~mm}$ were employed in $95,6 \%$ of the cases. The duration of the treatment was 24 months in $76,2 \%$ of the $B E S$. Esophageal perforations occurred in 6 patients (I,2\%), without mortality.

RESULTS. Were considered excellent, good and bad results, respectively in $76,2 \%$, $18,2 \%$ and $5,6 \%$ of the cases. On the other hand, excellent results were recorded in $81,0 \%$ of the peptic stenosis, $66,1 \%$ of the caustic stenosis and $82,7 \%$ of the anastomotic stenosis. The conservative treatment failed in $9,3 \%$ of the caustic stenosis, $4,3 \%$ of the anastomotic stenosis and 3,9\% of the peptic stenosis. Thus, the caustic stenosis were unsuccessfull in the highest percentage of unsuccessful.

CONCLUSION. The conservative treatment using guidewire dilators (Savary-Gilliard and Eder-Puestow) is the first choice in the BES, is effective for long time, with short complications and the surgical treatment is indicated only when the dilatations failed. [Rev Ass Med Brasil 200I; 47(3): 236-43]

KEY WORDS: Benign esophageal strictures. Esophageal dilatations. Esophageal dilators. Esophageal dilators. Esophagus.

\section{REFERÊNCIAS}

I. Earlam R, Cunha-MeloJR. Benign oesophageal strictures: historical and technical aspects of dilation. Brit J Surg 1981; 68:829-36.

2. Lanza FL, Graham DY. Bouginage is effective therapy for most benign esophageal strictures. JAMA 1978; 240:844-7.

3. Saeed ZA, Graham DY. Treatment of benign esophageal strictures. Where do we go from here? Dig Dis Sci 1994; 39:2099-101.

4. Wo JM, Waring JP. Medical therapy of gastroesophageal reflux and management of esophageal strictures. Surg Clin North Am 1997; 77: 1041-55.
5. Saeed ZA, Winchester CB, Ferro PS, Michaletz PA, Schwartz JT, Graham DY. Prospective randomized comparison of polyvinil bougies and through-the-scope balloons for dilation of peptic strictures of esophagus. Gastrointest Endosc 1995; 4 I: 1 89-95

6. Lahot D, Broor SL, Basu PP, Gupta A, Sharma R, Pant CS. Corrosive esophageal strictures: predictor of response to endoscopic dilation. Gastrointest Endosc 1995; 41:196-200.

7. Andreollo NA, Brandalise NA, Lopes LR, Callejas Neto F, Leonardi LS. Esophageal strictures after prolonged nasogastric intubation. ABCD Arq Bras Cir Dig 1987; 2:50-4.

8. Yararbai O, Osmanodlu H, Kaplan $\mathrm{H}$, Tokat $Y$, Coker A, Korkut M, et al. Esophagocoloplasty in the management of post corrosive strictures of the esophagus. Hepatogastroenterology 1998; 45:59-64.

9. Parkman HP, Ogorek CP, Harris AD, Cohen S. Non operative management o esophageal strictures following esophogomyotomy for achalasia. Dig Dis Sci 1994; 39:2 $102-8$.

10. Swaroop VS, Desai DC, Mohandas KM, Dhir V, Desai PB. Dilation of esophageal strictures induced by radiation therapy for cancer of esophagus. Gastrointest Endosc 1994; 40:3 I I -5.

II. El-Serag HB, Sonnenberg A. Association of esophagitis and esophageal strictures with diseases treated with nonsteroidal anti-inflammatory drug. Am J Gastroenterol 1996; 92:52-5.

12. Tan B-S, Kennedy C, Morgan R, Owen W, Adam A. Using uncovered metallic endoprotheses to treat recurrent benign esophageal strictures. AJR 1997; 169: | 28|-4.

13. Song H-Y, Park S, Do Y-S, Yoon H-K, Sung KB, Sohn $\mathrm{K}-\mathrm{H}$, et al. Expandable metallic stent placement in patients with benign esophageal stricture: Results of long-term follow-up. Radiology 1997; 203: 131-6.

14. American Society for Gastrointestinal Endoscopy. Esophageal dilation. Gastrointest Endosc 1998; 48:702-4.

15. Andreollo NA, Brandalise NA, Leonardi LS, Callejas Neto F. Tratamento das estenoses benignas do esôfago com o dilatador de EderPuestow. GED 1984; 3:9-12.

16. Saeed ZA, Ramirez FC, Hepps KS, Cole RA, Schneider FE, Ferro PS, et al. An objective end point for dilation improves out come of peptic esophageal strictures: a prospective randomized trial. Gastrointest Endosc 1997; 45:354-9

17. Broor SL, Raju GS, Bose PP, Lahoti D, Ramesh GN, Kumar A, et al. Long term results of endoscopic dilatation for corrosive oesophageal strictures. Gut 1993; 34: 1498-501.

18. Patterson DJ, Graham DY, Smith L, Schwartz JT, Alpert E, Lanza FL, et al. Natural history og benign esophageal stricture treated by dilation. Gastroenterology 1983; 85:346-50.

19. Dumon J-F, Meric B, Sivak MV, Fleischer D. A new method of esophageal dilation using Savary-Gilliard bougies. Gastrointest Endosc 1985; 31 : 379-82.
20. Cox JGC, Winter RK, Maslin SC. Balloon or bougie for dilation of benign esophageal stricture? Dig Dis Sci 1994; 39:774-81.

21. Wesdorp ICE, Bartelsman JFWM, Den Hartog Jager FCA, Huibregtse K, Tytgat GN. Results of conservative treatment of benign esophageal strictures: a follow-up study in 100 patients. Gastroenterology 1982; 82:487-93.

22. I Dhir V, Swaroop VS, Mohandas KM, Desai DC. Dilation of proximal esophageal strictures following therapy for head and neck cancer: experience with Savary-Gilliard dilator. J Surg Oncol 1996; 63: 187-90

23. Wu M-Ho, Lai W-W, Lin M-Y, Chou N-S. Prevention and management of strictures after hypopharyngocolostomy ou esophagocolostomy. Ann Thorac Surg 1994; 58: 108-II.

24. Heitmiller RF, Fischer A, Liddicoat JR. Cervical esophagogastric anastomosis: results following esophagectomy for carcinoma. Dis Esophagus 1999; 1 2:264-9

25. Ikeya T, Ohwada S, Ogawa T, Tanahashi Y, Takeyoshi I, Koyama T, et al. Endoscopic balloon dilation for benign esophageal anastomotic stricture: factors influencing its effectiveness. Hepatogastroenterology 1999; 46:959-66.

26. Garg PK, Sidhu SS, Bhargava DK. Role of omeprazole in prevention and treatment of posendoscopic variceal sclerotherapy esophageal complications. Dig Dis Sci, 1995; 40:1569-73.

27. Pereira-Lima JC, Ramires RP, Zamin I, Cassal AP, Marroni CA, Mattos AA. Endoscopic dilation of benign esophageal strictures: Report on 1043 procedures. Am J Gastroenterol 1999; 94:|497-50|.

28. Fleischer DE, Bejamin SB, Cattau EL. A marked guidewire facilities esophageal dilation. Am J Gastroenterol 1989; 84:359-61.

29. Silvius SE, Nebel O, Rogers G. Endoscopic complications. Results of the 1974 American Society of Gastrointestinal Endoscopy Survey. JAMA 1976; 235:928-30.

30. Duseja A, Chawla YK, Singh RP, Sharma TR, Kaur $U$, Dilawari JB Dilatation of benign oesophageal strictures: 10 years' experience with Celestin dilators. J Gastroenterol Hepatol 2000; 15:26-9.

31. Kadakia SC, Parker A, CarrougherJG. Esophageal dilation with polyvinil bougies using a marked guidewire without the aid of fluoroscopy: an update. Am J Gastroenterol 1993; 88: $1381-6$.

32. Jaspersen D, Diehl KL, Geyer P, Martens E, Arps $\mathrm{H}$ Benign proximal esophageal stenosismostly a complication of gastroesophageal reflux disease. Dtsch Med Wochenschr 1999; 124:205-8.

Artigo recebido: 13/09/2000

Aceito para publicação: 19/02/200। 